\title{
Local Synchronization of a Complex Network Model
}

\author{
Wenwu Yu, Student Member, IEEE, Jinde Cao, Senior Member, IEEE, Guanrong Chen, Fellow, IEEE, \\ Jinhu Lü, Senior Member, IEEE, Jian Han, Student Member, IEEE, and Wei Wei
}

\begin{abstract}
This paper introduces a novel complex network model to evaluate the reputation of virtual organizations. By using the Lyapunov function and linear matrix inequality approaches, the local synchronization of the proposed model is further investigated. Here, the local synchronization is defined by the inner synchronization within a group which does not mean the synchronization between different groups. Moreover, several sufficient conditions are derived to ensure the local synchronization of the proposed network model. Finally, several representative examples are given to show the effectiveness of the proposed methods and theories.
\end{abstract}

Index Terms-Complex network, linear matrix inequality, local synchronization, Lyapunov functional, reputation computation, trust, virtual organization.

\section{INTRODUCTION}

$\mathbf{T}$ HE SEMANTIC Web [32]-[34] strives for an intelligent Web searching and linking structures based on the metadata available in the Web. The Semantic Web may be viewed as an approach to distributed artificial intelligence, with tools to generate the metadata attached to Web pages and to reason about their significance, particularly when different resources can be combined and individual metadata can guide what to combine, as well as the significance of their combinations.

Manuscript received February 4, 2008; revised July 13, 2008 and July 30, 2008. First published December 9, 2008; current version published January 15, 2009. This work was supported in part by the National Basic Research (973) Program of China under Grant 2003CB317004 and Grant 2007CB310805, by the National Natural Science Foundation of China under Grant 60574043, Grant 60221301, and Grant 60772158, by the International Joint Project funded by NSFC and the Royal Society of the United Kingdom, by the Hong Kong Research Grants Council under CERG Grant CityU 1117/08E, by the NSFC-HKRGC Joint Research Scheme under Grant N-CityU107/07, by the Important Direction Project of Knowledge Innovation Program of the Chinese Academy of Sciences under Grant KJCX3-SYW-S01, and by the Scientific Research Startup Special Foundation on Excellent Ph.D. Thesis and Presidential Award of Chinese Academy of Sciences. This paper was recommended by Associate Editor J. Wang.

W. Yu is with the Department of Mathematics, Southeast University, Nanjing 210096, China, and also with the Department of Electronic Engineering, City University of Hong Kong, Kowloon, Hong Kong (e-mail: wenwuyu@gmail.com).

J. Cao is with the Department of Mathematics, Southeast University, Nanjing 210096, China (e-mail: jdcao@seu.edu.cn).

G. Chen is with the Department of Electronic Engineering, City University of Hong Kong, Kowloon, Hong Kong (e-mail: eegchen@ @ityu.edu.hk).

$\mathrm{J}$. Lü is with the Institute of Systems Science, Academy of Mathematics and Systems Science, Chinese Academy of Sciences, Beijing 100190, China (e-mail: jhlu@iss.ac.cn).

J. Han is with the School of Engineering and Applied Sciences, Harvard University, Cambridge, MA 02138 USA (e-mail: jianhan@fas.harvard.edu).

W. Wei is with the Department of Electrical Engineering, Stanford University, Stanford, CA 94305 USA (e-mail: weiwei1@ stanford.edu).

Color versions of one or more of the figures in this paper are available online at http://ieeexplore.ieee.org.

Digital Object Identifier 10.1109/TSMCB.2008.2004964
Combining services becomes more complex as one tries to match precise input and output service ports corresponding to a message-based remote procedure, called implementation in the Web or Grid service model. The Semantic Grid [24], [29] is similar to the Semantic Web, with the only difference is that the former shares resources in accordance with certain architectures and standard Grid infrastructures. The Semantic Grid allows anyone around the world to develop their own tools to interact with the Semantic Grid resources easily. Virtual Organizations [26], [27], which allow access to large computing resources, have become increasingly popular. The real and specific problem that underlies the Grid concept is coordinated with resource sharing and problem solving dynamically among multiinstitutional organizations. The sharing that we are concerned with is not primarily files exchange but rather direct access to computers, software, data, and other resources, as is required by a range of collaborative problem-solving and resource-brokering strategies emerging from industries, engineering, and technologies.

Authentication and authorization, i.e., deciding whether a user can have access to a certain resource, are essential in distributed applications. The Grid computing field can be characterized as a collection of heterogeneous computing resources that is shared by many individuals and organizations. This has given rise to the concept of Virtual Organization [26], [27]. A Virtual Organization is a collection of people in some administrative domain. The user can be a member of any internal group in an organization and has multiple roles in many organizations. The main problem in Virtual Organizations is the security, which includes how to identify a user and how to evaluate the functions that a user can perform.

In order to implement secure and reliable high-performance computing services, we study how to support the security infrastructure for Virtual Organizations. Security on Virtual Organizations [25], [28], [31] has attracted increasing attentions from various research communities in recent years, due to the unique ability of marshalling collections of heterogeneous computers and resources, enabling easy access to diverse resources and services that otherwise could not be possible without a good computational model. However, the concept of Virtual Organization introduces its own set of security challenges, as users and resource providers can come from mutually distributed administrative domains and any participant can behave maliciously.

Recently, reputation [31] has been recognized as an important factor for Virtual Organization security. However, no models have been proposed for integrating computational systems into Virtual Organizations. In this paper, we introduce a new complex network model for computing the reputation degrees of Virtual Organizations. 
Complex networks exist in all fields of sciences and humanities and have been intensively studied over the last few years [1]-[3], [35]-[50]. Among these are computer networks, the World Wide Web (WWW), telephone call graphs, food webs, biological neural networks, electrical power grids, coauthorship and citation networks of scientists, cellular and metabolic networks, etc. Many properties of real-world complex networks can be well understood by considering the network's internal interactions. It is now known that the basic properties of very large networks are mainly determined by the way the connections among the nodes are made. This motivates us to use a complex network model to study the reputation degrees of Virtual Organizations.

In the recent network studies to describe the transition from a regular to a random network, Watts and Strogatz [2], [30] introduced the so-called small-world network model. This model exhibits a high degree of clustering as in the regular networks and a small average distance among nodes as in the random networks. A common feature of such models is that the degree distribution $P(k)$ (defined as the probability that a randomly selected node has degree $k$, i.e., a node has $k$ connections) of the network peaks at an average value and decays exponentially. Such an exponential network is homogeneous in nature; each node has roughly the same number of connections.

On the other hand, it was discovered that many complex networks, such as the Internet, the WWW, and the metabolic network, are scale-free. This means that the degree distribution of these complex networks follow a power law form, $P(k) \sim k^{-\gamma}$ for a large $k$, where $\gamma$ is a positive constant determined by the network topology. Scale-free networks are inhomogeneous in nature, i.e., most nodes have very few connections but a small number of particular nodes have many connections. Interestingly, many complex networks are smallworld and also scale-free networks.

Many large-scale systems in nature and human societies, such as biological neural networks, ecosystems, metabolic pathways, the Internet, the WWW, electrical power grids, etc., can be described by networks, with the nodes representing individuals in the system and the edges representing the connections among them. A node in the network is a fundamental unit with detailed contents that can present an entity in the Semantic Grid. Very recently, Wang and Chen [3], [4] discussed a dynamical complex network model and also investigated its synchronization. A lot of research papers [7]-[17], [37]-[39], [44], [45] have been published based on this same model, mainly on the concerned issue of synchronization. In [35], we proposed a new complex network model for reputation computation in Virtual Organizations and also investigated its convergence dynamics. In this paper, we will further study this model and consider its local synchronization based on the results in [44] and the references therein. Here, the local synchronization is defined by the inner synchronization within a group where some nodes in the network can be synchronized while synchronization in the whole network cannot be achieved. The so-called local synchronization has rarely been investigated elsewhere and is still a challenging problem nowadays. The designed principles behind this phenomenon are very useful for theoretical works and future applications.

\section{BACKGROUND AND MODEL FORMULATION}

In order to derive a new model for reputation computation for Virtual Organizations, the concept of reputation [23] is introduced first.

When making trust-based decisions, an entity can rely on others with the information pertaining to them. For example, if entity $x$ wants to make a decision whether to have a transaction with entity $y$, which is unknown to $x$, then $x$ relies on the reputation of $y$. The definition of reputation that we will use was introduced in [23], as follows.

Definition 1: The reputation of an entity is an expectation of its behavior based on other entities' observations or the collective information about the entity's past behavior within a specific context at a given time.

In the following, we establish a new complex network model to study the reputation computation for Virtual Organizations to evaluate their reputations.

First, we review the coupled complex network model used in [3], [4], [7]-[20].

Consider a complex dynamical network consisting of $N$ linearly and diffusively coupled identical nodes, with each node being an $n$-dimensional dynamical system. The state equations of the network are

$$
\begin{array}{r}
\dot{x}_{i}(t)=f\left(x_{i}(t)\right)+c \sum_{j=1, j \neq i}^{N} G_{i j} \Gamma\left(x_{j}(t)-x_{i}(t)\right) \\
i=1,2, \ldots, N
\end{array}
$$

where $x_{i}(t)=\left(x_{i 1}(t), x_{i 2}(t), \ldots, x_{i n}(t)\right)^{\mathrm{T}} \in R^{n}$ represents the state vectors of node $i, i=1,2, \ldots, N ; f: R^{n} \longrightarrow R^{n}$ is continuously differentiable; the constant $c>0$ represents the coupling strength; $\Gamma=\operatorname{diag}\left(\gamma_{1}, \gamma_{2}, \ldots, \gamma_{n}\right) \in R^{n \times n}$ is a constant $0-1$ matrix linking the coupled variables; and $G=\left(G_{i j}\right)_{N \times N}$ is the coupling configuration matrix representing the topological structure of the network, in which $G_{i j}$ is defined as follows: If there is a connection between the nodes $i$ and $j(j \neq i)$, then the coupling strength $G_{i j}=G_{j i}=1$; otherwise, $G_{i j}=G_{j i}=0(j \neq i)$, and the diagonal elements of matrix $G$ are defined by

$$
G_{i i}=-\sum_{j=1, j \neq i}^{N} G_{i j}=-\sum_{j=1, j \neq i}^{N} G_{j i} .
$$

In this case, the network (1) can be written as

$$
\dot{x}_{i}(t)=f\left(x_{i}(t)\right)+c \sum_{j=1}^{N} G_{i j} \Gamma x_{j}(t), \quad i=1,2, \ldots, N .
$$

Hereafter, suppose that the network (3) is connected in the sense that there are no isolate clusters; hence, the coupling configuration matrix $G$ is an irreducible matrix.

The synchronization of complex networks has been intensively investigated [7]-[17], [37]-[39] based on the model (1) or (3). Although the model (3) reflects the complexity from the network structure, it is a simple uniform dynamical network without time-varying or delayed coupling. Considering that 
there are usually some time delays in influence and response due to the finite speeds of transmission and spreading, as well as traffic congestions, time delays should be modeled in order to simulate more realistic networks. Li and Chen [15] discussed the following complex dynamical network model with delayed coupling:

$\dot{x}_{i}(t)=f\left(x_{i}(t)\right)+c \sum_{j=1}^{N} G_{i j} \Gamma x_{j}(t-\tau), \quad i=1,2, \ldots, N$

where $\tau$ is the time delay. In [7], [8], and [11], the following system was studied:

$$
\begin{aligned}
\dot{x}_{i}(t)=- & C x_{i}(t)+A f\left(x_{i}(t)\right)+B f\left(x_{i}(t-\tau)\right) \\
& +I(t)+c \sum_{j=1}^{N} G_{i j} \Gamma x_{j}(t), \quad i=1,2, \ldots, N
\end{aligned}
$$

where $C=\operatorname{diag}\left(c_{1}, c_{2}, \ldots, c_{n}\right) \in R^{n \times n}$ is a diagonal matrix with positive diagonal entries $c_{i}>0 ; i=1,2, \ldots, n ; A=$ $\left(a_{i j}\right)_{n \times n}$ and $B=\left(b_{i j}\right)_{n \times n}$ are the weight and delayed weight matrices, respectively; $I(t)=\left(I_{1}(t), I_{2}(t), \ldots, I_{n}(t)\right)^{\mathrm{T}} \in R^{n}$ is an external input vector; $\Gamma=\operatorname{diag}\left(\gamma_{1}, \gamma_{2}, \ldots, \gamma_{n}\right) \in R^{n \times n}$; and $f\left(x_{i}(t)\right)=\left(f_{1}\left(x_{i 1}(t)\right), f_{2}\left(x_{i 2}(t)\right), \ldots, f_{n}\left(x_{i n}(t)\right)\right)^{\mathrm{T}} \in R^{n}$ corresponds to the activation functions of the nodes. Equivalently, the system (5) can be written as

$$
\begin{aligned}
\dot{x}_{i k}(t)= & -c_{k} x_{i k}(t)+\sum_{l=1}^{n} a_{k l} f_{l}\left(x_{i l}(t)\right) \\
& +\sum_{l=1}^{n} b_{k l} f_{l}\left(x_{i l}(t-\tau)\right)+I_{k}(t) \\
& +c \sum_{j=1}^{N} G_{i j} \gamma_{k} x_{j k}(t) \\
i= & 1,2, \ldots, N ; \quad k=1,2, \ldots, n .
\end{aligned}
$$

In this paper, the proposed complex network model in the reputation computation for Virtual Organizations [35] is further investigated. Consider an array of $N$ linearly connected complex network model in the following form:

$$
\begin{aligned}
\dot{x}_{i}(t)= & -C x_{i}(t)+A f\left(x_{i}(t)\right) \\
+ & +B \int_{-\infty}^{t} K(t-s) f\left(x_{i}(s)\right) d s+I_{i}(t) \\
+ & \sum_{j=1, j \neq i}^{N} G_{i j}\left[\Gamma\left(x_{j}(t)-x_{i}(t)\right)\right. \\
& +\Lambda\left(\int_{-\infty}^{t} K(t-s) x_{j}(s) d s\right. \\
& \left.\left.-\int_{-\infty}^{t} K(t-s) x_{i}(s) d s\right)\right]
\end{aligned}
$$

or, equivalently

$$
\begin{aligned}
\dot{x}_{i k}(t)= & -c_{k} x_{i k}(t)+\sum_{l=1}^{n} a_{k l} f_{l}\left(x_{i l}(t)\right) \\
& +\sum_{l=1}^{n} b_{k l} \int_{-\infty}^{t} K_{l}(t-s) f_{l}\left(x_{i l}(s)\right) d s+I_{i k}(t) \\
& +\sum_{j=1}^{N} G_{i j}\left[\gamma_{k}\left(x_{j k}(t)-x_{i k}(t)\right)\right. \\
& +\lambda_{k}\left(\int_{-\infty}^{t} K_{k}(t-s) x_{j k}(s) d s\right. \\
& \left.\left.\quad \int_{-\infty}^{t} K_{k}(t-s) x_{i k}(s) d s\right)\right]
\end{aligned}
$$

where $\quad x_{i}(t)=\left(x_{i 1}(t), x_{i 2}(t), \ldots, x_{i n}(t)\right)^{\mathrm{T}} \in R^{n} \quad$ is $\quad$ the state vector of the $i$ th node of the network; $i=1,2, \ldots, N$; $C=\operatorname{diag}\left(c_{1}, c_{2}, \ldots, c_{n}\right) \in R^{n \times n}$ is a diagonal matrix with positive diagonal entries $c_{i}>0 ; \quad i=1,2, \ldots, n ; \quad A=$ $\left(a_{i j}\right)_{n \times n}$ and $B=\left(b_{i j}\right)_{n \times n}$ are the weight and delayed weight matrices, respectively; $f\left(x_{i}(t)\right)=\left(f_{1}\left(x_{i 1}(t)\right)\right.$, $\left.f_{2}\left(x_{i 2}(t)\right), \ldots, f_{n}\left(x_{i n}(t)\right)\right)^{\mathrm{T}} \in R^{n} \quad$ represents the output of the node $i ; I_{i}(t)=\left(I_{i 1}(t), I_{i 2}(t), \ldots, I_{i n}(t)\right)^{\mathrm{T}} \in R^{n}$ is the external input vector; $\Gamma=\operatorname{diag}\left(\gamma_{1}, \gamma_{2}, \ldots, \gamma_{n}\right) \in R^{n}$ and $\Lambda=\operatorname{diag}\left(\lambda_{1}, \lambda_{2}, \ldots, \lambda_{n}\right) \in R^{n}$ are diagonal matrices, which implies that the $r$ th state variable of the $i$ th node of the network is only affected by the $r$ th state variables of the other nodes of the network; the weight function $K(t)=\operatorname{diag}\left(K_{1}(t), K_{2}(t), \ldots, K_{n}(t)\right) \in R^{n \times n}$ is a nonnegative bounded function defined on $[0,+\infty)$ that reflects the influence of the past states on the current dynamics; and $G=\left(G_{i j}\right)_{N \times N}$ is the coupling configuration matrix representing the coupling strengths and topological structure of the network, in which $G_{i j}$ is defined as follows: If there is a connection between the nodes $i$ and $j(j \neq i)$, then the coupling strength $G_{i j}>0$; otherwise, $G_{i j}=0$, and the diagonal elements of matrix $G$ are defined similarly as (2) by

$$
G_{i i}=-\sum_{j=1, j \neq i}^{N} G_{i j} .
$$

The coupled complex network model (7) can be rewritten as

$$
\begin{aligned}
\dot{x}_{i}(t)= & -C x_{i}(t)+A f\left(x_{i}(t)\right) \\
& +B \int_{-\infty}^{t} K(t-s) f\left(x_{i}(s)\right) d s+I_{i}(t) \\
& +\sum_{j=1}^{N} G_{i j}\left[\begin{array}{c}
\left.\Gamma x_{j}(t)+\Lambda \int_{-\infty}^{t} K(t-s) x_{j}(s) d s\right] \\
i=1,2, \ldots, N
\end{array}\right.
\end{aligned}
$$


or, equivalently

$$
\begin{aligned}
\dot{x}_{i k}(t)= & -c_{k} x_{i k}(t)+\sum_{l=1}^{n} a_{k l} f_{l}\left(x_{i l}(t)\right) \\
& +\sum_{l=1}^{n} b_{k l} \int_{-\infty}^{t} K_{l}(t-s) f_{l}\left(x_{i l}(s)\right) d s+I_{i k}(t) \\
& +\sum_{j=1}^{N} G_{i j}\left[\gamma_{k} x_{j k}(t)+\lambda_{k} \int_{-\infty}^{t} K_{k}(t-s) x_{j k}(s) d s\right] \\
& \quad i=1,2, \ldots, N ; \quad k=1,2, \ldots, n .
\end{aligned}
$$

Next, we show that the complex model (7) can describe the dynamical evolution of the reputation for Virtual Organizations.

Let $x_{i}(t)$ denote the reputation degree of the $i$ th entity at time $t ; x_{i j}(t)(j=1,2, \ldots, n)$ be the $j$ th index of the reputation degree $x_{i}(t)$; and $C, A$, and $B$ be the inner coupling matrices, where $C$ is the restraint of the reputation degree, $A$ is the weight matrix representing the influence of the present reputation degree, and $B$ is the weight matrix representing the influence of the past reputation degree. Moreover, let $I$ be the external influence, the coupling configuration $G$ describe the interactions between pairs of entities, $\Gamma$ be the weight matrix of the influence of other entities' reputation degrees compared with a certain entity's own reputation degree at the present time, and $\Lambda$ be the weight matrix of the influence of other entities' past reputation degrees compared with a certain entity's own past reputation degree; assume that $\Gamma$ and $\Lambda$ are diagonal matrices for simplicity, which implies that each entity is mostly influenced by the other entities with the same indexes.

From the model (7), it is easy to see that the reputation degree that the entity $i$ holds is based on its own present and past reputation degrees and the relationships with other entities, as well as their present and past reputation degrees.

Now, define some special kernel matrix function $K(\cdot)$. If

$$
K_{i}(s)=\delta(s-\tau), \quad \tau>0, \quad i=1,2, \ldots, n
$$

where $\delta(\cdot)$ is the Dirac function, then the system (10) can be written as

$$
\begin{array}{r}
\dot{x}_{i}(t)=-C x_{i}(t)+A f\left(x_{i}(t)\right)+B f\left(x_{i}(t-\tau)\right) \\
+I_{i}(t)+\sum_{j=1}^{N} G_{i j}\left[\Gamma x_{j}(t)+\Lambda x_{j}(t-\tau)\right] \\
i=1,2, \ldots, N
\end{array}
$$

which is a model of delayed diffusively coupled complex networks. If

$$
K_{i}(s)=\left\{\begin{array}{ll}
0, & s \geq \tau \\
1, & s<\tau,
\end{array} \quad i=1,2, \ldots, n\right.
$$

where $\tau$ is a positive constant, then the system (10) can be written as

$$
\begin{aligned}
& \dot{x}_{i}(t)=-C x_{i}(t)+A f\left(x_{i}(t)\right) \\
&+B \int_{t-\tau}^{t} K(t-s) f\left(x_{i}(s)\right) d s+I_{i}(t) \\
&+\sum_{j=1}^{N} G_{i j}\left[\Gamma x_{j}(t)+\Lambda \int_{t-\tau}^{t} K(t-s) x_{j}(s) d s\right] \\
& i=1,2, \ldots, N
\end{aligned}
$$

which means that the long-term reputation degree of an entity is not considered. Moreover, $K_{i}(\cdot)$ could be a decaying function, which is decreasing with time, as

$$
K_{i}(s)=\alpha e^{-\alpha s}, \quad \alpha>0, \quad i=1,2, \ldots, n
$$

which satisfies

$$
\int_{o}^{+\infty} K_{i}(s) d s=1, \quad i=1,2, \ldots, n
$$

and it indicates that the reputation degree of the entity is mostly influenced by the more recent reputation degree.

Remark 1: In this paper, we study the reputation computation model (15) that satisfies (16). There are a lot of dynamical phenomena emerging from the complex model (15), such as stability, synchronization, bifurcation, and chaos. As a start on this new model, we only consider some simple phenomena in this paper. The stability of the model (15) implies that all the reputation degrees of entities tend to converge to some constants, which was studied in [35]. On the other hand, chaos means that the reputation degrees of entities are random and changeable, which will be studied elsewhere. In this paper, we investigate the local synchronization of the coupled complex network model (15). The local synchronization here means that each group of reputation degrees synchronizes with one another but that the whole network may not synchronize globally.

If the coupled system involves more than two nodes (chaotic oscillators), a variety of new phenomena can occur, including partial synchronization [18]-[20], which is referred to as local synchronization in this paper. A state of local synchronization is said to occur when the interacting oscillators synchronize with one another in each group; however, there may not be synchrony among the groups. When the coupling matrix $G$ is irreducible and symmetric, with all the off-diagonal elements being nonnegative and satisfying (2), a complete synchronization analysis has been studied, e.g., in [7]-[17]. Much less is understood, however, on local synchronization that occurs in diffusively coupled networks. In [18]-[20], some simple low dimensional coupled models without discrete or distributed time delays were considered. The method used in those papers cannot be used to study the model (15) here. In this paper, we study the local synchronization of the model (15) based on the synchronization manifold method proposed mainly in our paper [44] and the references therein. 
First, the following assumptions are imposed.

$\mathrm{A}_{1}: f_{i}\left(x_{i}\right)(i=1,2, \ldots, n)$ is monotonically nondecreasing on $R$.

$\mathrm{A}_{2}: f_{i}(\cdot)(i=1,2, \ldots, n)$ is Lipschitz continuous, i.e., there exist constants $F_{i}$, such that

$$
\left|f_{i}(x)-f_{i}(y)\right| \leq F_{i}|x-y| \quad \forall x, y \in R .
$$

$\mathrm{A}_{3}$ : The coupling matrix $G$ is symmetric and diffusive, namely, satisfying

$$
\begin{array}{rlrl}
G_{i j} & \geq 0, & i \neq j \\
G_{i i} & =-\sum_{j=1, j \neq i}^{N} G_{i j}, & & i=1,2, \ldots, N .
\end{array}
$$

$\mathrm{A}_{4}$ : Let $m \leq N$ and the coupling matrix $G=\left(\begin{array}{cc}N_{1} & N_{2} \\ N_{2}^{\mathrm{T}} & N_{3}\end{array}\right)$, where $\quad N_{1} \in R^{m \times m} ; \quad N_{2} \in R^{m \times(N-m)} ; \quad N_{3} \in$ $R^{(N-m) \times(N-m)}$; and assume that all rows in $N_{2}$ are the same, i.e., $N_{2}=(u, u, \ldots, u)^{\mathrm{T}}$, and that $u=\left(u_{1}, u_{2}, \ldots, u_{N-m}\right)^{\mathrm{T}}$ is a vector. Moreover, $I_{1}(t)=I_{2}(t)=\cdots=I_{m}(t)$.

Remark 2: In this paper, we study the local synchronization of the first $m$ nodes in the coupled network model (15) for simplicity. For a general case, we can use a transformation to convert the study to the local synchronization of the first $m$ nodes. Note that when Assumption $\mathrm{A}_{4}$ is satisfied, it indicates that the first $m$ nodes of the coupled network have the same connections with the other $N-m$ nodes. They more likely belong to the same group and have the same evolvement of reputation degrees. Thus, without loss of generality, we only study the local synchronization of the first $m$ nodes in the model (15).

Assume that the system (15) satisfies the following initial conditions. $\quad x_{i}(t)=\phi_{i}(t) \in \mathcal{C}([-\tau, 0], R) \quad(i=1,2, \ldots, N)$, where $\mathcal{C}([-\tau, 0], R)$ denotes the set of all continuous functions from $[-\tau, 0]$ to $R$.

Next, some basic definitions and lemmas are needed, which are very much the same as those given in [5], [7]-[9], and [11].

Definition 2: The set $\mathbf{S}=\left\{x=\left(x_{1}(s), x_{2}(s), \ldots, x_{N}(s)\right)\right.$ : $\left.x_{i}(s) \in \mathcal{C}([-\tau, 0], R), x_{i}(s)=x_{j}(s), i, j=1,2, \ldots, N\right\} \quad$ is called the global synchronization manifold.

Definition 2A: The set $\mathbf{S}^{\prime}=\left\{x=\left(x_{1}(s), x_{2}(s), \ldots\right.\right.$, $\left.x_{N}(s)\right): x_{i}(s) \in \mathcal{C}([-\tau, 0], R), x_{i}(s)=x_{j}(s), i, j=1,2$, $\ldots, m\}$ is called the local synchronization manifold.

Definition 3: The local synchronization manifold $\mathbf{S}^{\prime}$ is said to be locally asymptotically stable, or equivalently, the coupled system (15) is locally asymptotically synchronized if, for all $\varepsilon>0$, there exists $\delta>0$, such that if $\sup _{s \in[-\tau, 0]} \| \phi_{i}(s)-$ $\phi_{j}(s) \| \leq \delta$, then for a sufficiently large $T>0$, there holds

$$
\left\|x_{i}(t)-x_{j}(t)\right\| \leq \varepsilon
$$

for all $t>T, i, j=1,2, \ldots, m$.

Definition 4: Let $\widehat{R}$ denote a ring, and define $T(\widehat{R}, K)=$ \{the set of matrices with entries $\widehat{R}$ such that the sum of the entries in each row is equal to $K$ for some $K \in \widehat{R}$ \}.

Definition 5: The set of $M_{1}^{N}(1): M_{1}^{N}(1)$ is composed of matrices with $N$ columns; each row (for instance, the $i$ th row) of $\widetilde{M} \in M_{1}^{N}(1)$ has exactly one entry of $\alpha_{i}$ and one entry of $-\alpha_{i}$, where $\alpha_{i} \neq 0$ and all other entries are zeros.

Definition 5A: The set of $M_{1}^{N, m}(1): M_{1}^{N}(1)$ is composed of matrices with $N$ columns; each row (for instance, the $i$ th row) of $\widetilde{M} \in M_{1}^{N}(1)$ has exactly one entry of $\alpha_{i}$ and one entry of $-\alpha_{i}$ in the first $m$ columns, where $\alpha_{i} \neq 0$ and all other entries are zeros.

Definition 6: The set of $M_{1}^{N}(n)$ are matrices $\mathbf{M}$ obtained by replacing entry $m_{i j}$ in $\widetilde{M} \in M_{1}^{N}(1)$ with $m_{i j} E_{n}$, where $E_{n}$ is the $n$-dimensional identity matrix, i.e., $M_{1}^{N}(n)=$ $\left\{\mathbf{M}=\widetilde{M} \otimes E_{n}: \widetilde{M} \in M_{1}^{N}(1)\right.$ and $E_{n}$ is the $n$-dimensional identity matrix \}, where $\otimes$ is the Kronecker product.

Definition 6A: The set of $M_{1}^{N, m}(n)$ represents the matrices $\mathbf{M}$ obtained by replacing the entry $m_{i j}$ in $\widetilde{M} \in M_{1}^{N, m}(1)$ with $m_{i j} E_{n}$, where $E_{n}$ is the $n$-dimensional identity matrix, i.e., $\quad M_{1}^{N}(n)=\left\{\mathbf{M}=\widetilde{M} \otimes E_{n}: \widetilde{M} \in\right.$ $M_{1}^{N, m}(1)$ and $E_{n}$ is the $n$-dimensional identity matrix $\}$, where $\otimes$ is the Kronecker product.

Definition 7: $M_{2}^{N}(n) \subset M_{1}^{N}(n)$. If $M \in M_{2}^{N}(n)$, then for any pair of indices $i$ and $j$, there exist indices $j_{1}, j_{2}, \ldots, j_{l}$, where $j_{1}=i$ and $j_{l}=j$, and $p_{1}, p_{2}, \ldots, p_{l-1}$, such that $M_{p_{q}, j_{q}} \neq 0$ and $M_{p_{q}, j_{q+1}} \neq 0$ for all $1 \leq q<l$.

Definition 7A: $M_{2}^{N, m}(n) \subset M_{1}^{N, m}(n)$. If $M \in M_{2}^{N, m}(n)$, then for any pair of indices $i$ and $j(i, j \leq m)$, there exist indices $j_{1}, j_{2}, \ldots, j_{l}$, where $j_{1}=i$ and $j_{l}=j$, and $p_{1}, p_{2}, \ldots, p_{l-1}$, such that $M_{p_{q}, i_{q}} \neq 0$ and $M_{p_{q}, i_{q+1}} \neq 0$ for all $1 \leq q<l$.

Lemma 1 [5]: Let $G$ be an $N \times N$ matrix in $T(\widehat{R}, K)$. Then, the $(N-1) \times(N-1)$ matrix $H$ defined by $H=M G J$ satisfying $M G=H M$ is the $(N-1) \times N$ matrix

$$
M=\left(\begin{array}{cccccc}
1 & -1 & & & & \\
& 1 & -1 & & & \\
& & & \ddots & & \\
& & & & 1 & -1
\end{array}\right)
$$

where $J$ is the $N \times(N-1)$ matrix

$$
J=\left(\begin{array}{ccccc}
1 & 1 & 1 & \cdots & 1 \\
0 & 1 & 1 & \cdots & 1 \\
& & \ddots & & 1 \\
& & \cdots & 1 & 1 \\
0 & 0 & \cdots & 0 & 1 \\
0 & 0 & 0 & \cdots & 0
\end{array}\right)
$$

in which 1 is the multiplicative identity of $\widehat{R}$. The matrix $H$ can be written explicitly as $H_{(i, j)}=\Sigma_{k=1}^{j} G_{(i, k)}-G_{(i+1, k)}$, for $i, j \in\{1,2, \ldots, N-1\}$.

Lemma 2: Under Assumption $\mathrm{A}_{4}$, the $(m-1) \times(m-1)$ matrix $\widetilde{H}$ defined by $\widetilde{H}=M N_{1} J$ satisfying $\widetilde{M} G=\widetilde{H} \widetilde{M}$ is the $(m-1) \times N$ matrix

$$
\widetilde{M}=\left(\begin{array}{ccccccccc}
1 & -1 & & & & & 0 & \cdots & 0 \\
& 1 & -1 & & & & 0 & \cdots & 0 \\
& & & \ddots & & & 0 & \cdots & 0 \\
& & & & 1 & -1 & 0 & \cdots & 0
\end{array}\right)=(M
$$


where $M \in R^{(m-1) \times m}, O \in R^{(m-1) \times(N-m)}$, and each entry of $O$ is zero and where $\widetilde{J}$ is the $N \times(m-1)$ matrix

$$
\widetilde{J}=\left(\begin{array}{ccccc}
1 & 1 & 1 & \cdots & 1 \\
0 & 1 & 1 & \cdots & 1 \\
& & \ddots & & 1 \\
& & \cdots & 1 & 1 \\
0 & 0 & \cdots & 0 & 1 \\
0 & 0 & 0 & \cdots & 0 \\
\cdots & \cdots & & \cdots & 0 \\
0 & 0 & 0 & \cdots & 0
\end{array}\right)=\left(\begin{array}{c}
J \\
O^{\mathrm{T}}
\end{array}\right)
$$

where $J \in R^{m \times(m-1)}$. Moreover, $M$ and $J$ are similar, as defined in (21) and (22).

Proof:

$$
\widetilde{M} G=\left(\begin{array}{ll}
M & O
\end{array}\right)\left(\begin{array}{ll}
N_{1} & N_{2} \\
N_{2}^{\mathrm{T}} & N_{3}
\end{array}\right)=\left(\begin{array}{ll}
M N_{1} & M N_{2}
\end{array}\right) .
$$

Under Assumption $\mathrm{A}_{4}$, each row in $N_{2}$ is the same; thus, we obtain

$$
M N_{2}=O
$$

Moreover, $N_{1}$ is an $m \times m$ matrix in $T(\widehat{R}, K)$. From Lemma 1, we have

$$
M N_{1}=M N_{1} J M
$$

thus

$$
\widetilde{M} G=\left(\begin{array}{ll}
M N_{1} J M & O
\end{array}\right)=M N_{1} J(M \quad O)=M N_{1} J \widetilde{M} .
$$

This completes the proof.

Lemma 3 [5]: Let $x=\left(x_{1}, x_{2}, \ldots, x_{N}\right)^{\mathrm{T}}$, where $x_{i} \in R^{n}$, $i=1,2, \ldots, N$. Then, $x \in \mathbf{S}$ if and only if

$$
\|\mathbf{M} x\|=0
$$

holds for some $\mathbf{M} \in M_{2}^{N}(n)$.

We use $d(x)$ to denote a nonnegative real-valued function that measures the distance between two nodes. In particular, we define $d(x)$ to be in the following form:

$$
d(x)=\|\mathbf{M} x\|^{2}=x^{\mathrm{T}} \mathbf{M}^{\mathrm{T}} \mathbf{M} x, \quad \mathbf{M} \in M_{2}^{N}(n) .
$$

Considering the assumptions on $\mathbf{M}$, a crucial property of $d(x)$ is that $d(x) \longrightarrow 0$ if and only if $\left\|x_{i}(t)-x_{j}(t)\right\| \longrightarrow 0$ for all $i, j=1,2, \ldots, N$.

Lemma 4: Let $x=\left(x_{1}, x_{2}, \ldots, x_{N}\right)^{\mathrm{T}}$, where $x_{i} \in R^{n}$, $i=1,2, \ldots, N$. Then, $x \in \mathbf{S}^{\prime}$ if and only if

$$
\|\mathbf{M} x\|=0
$$

holds for some $\mathbf{M} \in M_{2}^{N, m}(n)$. We use $d(x)$ to denote a nonnegative real-valued function that measures the distance between two nodes. In particular, we define $d(x)$ to be in the following form:

$$
d(x)=\|\mathbf{M} x\|^{2}=x^{\mathrm{T}} \mathbf{M}^{\mathrm{T}} \mathbf{M} x, \quad \mathbf{M} \in M_{2}^{N, m}(n) .
$$

Considering the assumptions on $\mathbf{M}$, the crucial property of $d(x)$ is that $d(x) \longrightarrow 0$ if and only if $\left\|x_{i}(t)-x_{j}(t)\right\| \longrightarrow 0$ for all $i, j=1,2, \ldots, m$.

Lemma 5 [21]: By the definition of the Kronecker product, the following properties are satisfied in appropriate dimensions.

1) $(\alpha A) \otimes B=A \otimes(\alpha B)$

2) $(A+B) \otimes C=A \otimes C+B \otimes C$.

3) $(A \otimes B)(C \otimes D)=(A C) \otimes(B D)$.

Lemma 6 (Jensen Inequality [22]): For any constant matrix $W \in R^{m \times m}$ satisfying $W=W^{\mathrm{T}}$, scalar $r>0$, and vector function $\omega:[0, r] \in R^{m \times m}$ such that the integrations concerned are well defined, we have

$$
r \int_{0}^{r} \omega(s)^{\mathrm{T}} W \omega(s) d s \geq\left(\int_{0}^{r} \omega(s) d s\right)^{\mathrm{T}} W\left(\int_{0}^{r} \omega(s) d s\right) .
$$

Throughout this paper, for real symmetric matrices $P$ and $Q$, the notation $P \geq Q$ (respectively, $P>Q$ ) means that the matrix $P-Q$ is positive semidefinite (respectively, positive definite).

\section{LOCAL SYNCHRONIZATION OF THE COMPLEX NETWORK MODEL}

In this section, new criteria are derived for the local synchronization of the first $m$ nodes in the coupled system (15).

Let

$$
\begin{aligned}
\mathbf{C} & =E_{N} \otimes C, \quad \mathbf{C}^{\mathbf{1}}=E_{m-1} \otimes C \\
\mathbf{A} & =E_{N} \otimes A, \quad \mathbf{A}^{\mathbf{1}}=E_{m-1} \otimes A \\
\mathbf{\Gamma} & =G \otimes \Gamma \\
\mathbf{B} & =E_{N} \otimes B, \quad \mathbf{B}^{\mathbf{1}}=E_{m-1} \otimes B \\
\mathbf{K} & =E_{N} \otimes K, \quad \mathbf{K}^{\mathbf{1}}=E_{m-1} \otimes K \\
\mathbf{\Lambda} & =G \otimes \Lambda \\
x_{i}(t) & =\left(x_{i 1}(t), x_{i 2}(t), \ldots, x_{i n}(t)\right)^{\mathrm{T}} \quad \forall i=1,2, \ldots, N \\
x(t) & =\left(x_{1}^{\mathrm{T}}(t), x_{2}^{\mathrm{T}}(t), \ldots, x_{N}^{\mathrm{T}}(t)\right)^{\mathrm{T}} \\
\mathbf{f}(x(t)) & =\left(f^{\mathrm{T}}\left(x_{1}(t)\right), f^{\mathrm{T}}\left(x_{2}(t)\right), \ldots, f^{\mathrm{T}}\left(x_{N}(t)\right)\right)^{\mathrm{T}} \\
\mathbf{I}(t) & =\left(I_{1}^{\mathrm{T}}(t), I_{2}^{\mathrm{T}}(t), \ldots, I_{N}^{\mathrm{T}}(t)\right)^{\mathrm{T}} .
\end{aligned}
$$

The linearly coupled dynamical system (15) can be rewritten as

$$
\begin{gathered}
\dot{x}(t)=-\mathbf{C} x(t)+\mathbf{A f}(x(t))+\mathbf{B} \int_{t-\tau}^{t} \mathbf{K}(t-s) \mathbf{f}(x(s)) d s \\
+\mathbf{I}(t)+\boldsymbol{\Gamma} x(t)+\boldsymbol{\Lambda} \int_{t-\tau}^{t} \mathbf{K}(t-s) x(s) d s \\
i=1,2, \ldots, N .
\end{gathered}
$$

Theorem 1: Under Assumptions $\mathrm{A}_{2}-\mathrm{A}_{4}$, the dynamical system (29) is locally synchronized if there are positive definite matrices $\mathbf{P}=\left(p_{i j}\right) \in R^{(m-1) n \times(m-1) n}, \mathbf{Q}=\left(q_{i j}\right) \in$ $R^{(m-1) n \times(m-1) n}, \quad$ and $\quad \mathbf{R}=\left(r_{i j}\right) \in R^{(m-1) n \times(m-1) n}$ and 
a positive definite diagonal matrix $\boldsymbol{\Sigma}=\operatorname{diag}\left(\Sigma_{1}, \Sigma_{2}, \ldots\right.$, $\left.\Sigma_{(m-1) n}\right) \in R^{(m-1) n \times(m-1) n}$, such that

$\boldsymbol{\Omega}=\left(\begin{array}{cccc}\Xi^{\Xi} & \mathbf{P} \overline{\mathbf{H}} & \mathbf{P A}^{\mathbf{1}} & \mathbf{P B}^{\mathbf{1}} \\ \overline{\mathbf{H}}^{\mathrm{T}} \mathbf{P} & -\mathbf{R} & 0 & 0 \\ \mathbf{A}^{\mathbf{1}^{\mathrm{T}}} \mathbf{P} & 0 & \tau^{2} \mathbf{K}^{\mathbf{1}^{\mathrm{T}}}(0) \mathbf{Q} \mathbf{K}^{\mathbf{1}}(0)-\mathbf{\Sigma} & 0 \\ \mathbf{B}^{\mathbf{1}^{\mathrm{T}}} \mathbf{P} & 0 & 0 & -\mathbf{Q}\end{array}\right)<0$

where $\quad \Xi=-\mathbf{P C}^{\mathbf{1}}-\mathbf{C}^{\mathbf{1}^{\mathrm{T}}} \mathbf{P}+\mathbf{P H}+\mathbf{H}^{\mathrm{T}} \mathbf{P}+\mathbf{F} \boldsymbol{\Sigma} \mathbf{F}+$ $\tau^{2} \mathbf{K}^{\mathbf{1}^{\mathrm{T}}}(0) \mathbf{R K}^{\mathbf{1}}(0), \quad F=\operatorname{diag}\left(F_{1}, F_{2}, \ldots, F_{n}\right) \in R^{n \times n}$, $\mathbf{F}=E_{m-1} \otimes F, \quad \mathbf{H}=\left(M N_{1} J\right) \otimes \Gamma, \quad \overline{\mathbf{H}}=\left(M N_{1} J\right) \otimes \Lambda$, $M \in R^{(m-1) \times m}$, and $J \in R^{m \times(m-1)}$ have the same structures as those in (22) and (23).

Proof: Consider the following Lyapunov functional:

$$
V(t)=\sum_{i=1}^{3} V_{i}(t)
$$

where

$$
\begin{aligned}
V_{1}(t)= & x^{\mathrm{T}}(t) \mathbf{M}^{\mathrm{T}} \mathbf{P} \mathbf{M} x(t) \\
V_{2}(t)= & \tau \int_{-\tau}^{0} \int_{t+s}^{t}(\mathbf{M K}(t-\theta) \mathbf{f}(x(\theta)))^{\mathrm{T}} \mathbf{Q} \\
& \times(\mathbf{M K}(t-\theta) \mathbf{f}(x(\theta))) d \theta d s \\
V_{3}(t)= & \tau \int_{-\tau}^{0} \int_{t+s}^{t}(\mathbf{M K}(t-\theta) x(\theta))^{\mathrm{T}} \mathbf{R} \\
& \times(\mathbf{M K}(t-\theta) x(\theta)) d \theta d s .
\end{aligned}
$$

By calculating its derivative along the positive half trajectory of the system (29), we obtain

$$
\begin{aligned}
& \left.\dot{V}_{1}(t)\right|_{(29)}=2 x^{\mathrm{T}}(t) \mathbf{M}^{\mathrm{T}} \mathbf{P} \mathbf{M} \dot{x}(t) \\
& \leq 2 x^{\mathrm{T}}(t) \mathbf{M}^{\mathrm{T}} \mathbf{P} \mathbf{M} \\
& \times[-\mathbf{C} x(t)+\mathbf{A f}(x(t)) \\
& +\mathbf{B} \int_{t-\tau}^{t} \mathbf{K}(t-s) \mathbf{f}(x(s)) d s+\mathbf{I}(t) \\
& \left.+\boldsymbol{\Gamma} x(t)+\boldsymbol{\Lambda} \int_{t-\tau}^{t} \mathbf{K}(t-s) x(s) d s\right] .
\end{aligned}
$$

By the structure of $\mathbf{M}$ and Assumption $\mathrm{A}_{4}$, the following equalities are easy to verify:

$$
\begin{aligned}
& \mathbf{M C}=\mathbf{C}^{\mathbf{1}} \mathbf{M}, \quad \mathbf{M A}=\mathbf{A}^{\mathbf{1}} \mathbf{M}, \quad \mathbf{M B}=\mathbf{B}^{1} \mathbf{M} \\
& \mathbf{M K}=\mathbf{K}^{\mathbf{1}} \mathbf{M}, \quad \mathbf{M I}(t)=0 .
\end{aligned}
$$

Therefore, we obtain

$$
\begin{aligned}
& \left.\dot{V}_{1}(t)\right|_{(29)} \leq 2 x^{\mathrm{T}}(t) \mathbf{M}^{\mathrm{T}} \mathbf{P} \\
& \times\left[-\mathbf{C}^{\mathbf{1}} \mathbf{M} x(t)+\mathbf{A}^{\mathbf{1}} \mathbf{M f}(x(t))\right. \\
& +\mathbf{B}^{\mathbf{1}} \mathbf{M} \int_{t-\tau}^{t} \mathbf{K}(t-s) \mathbf{f}(x(s)) d s+\mathbf{M} \boldsymbol{\Gamma} x(t) \\
& \left.+\mathbf{M} \boldsymbol{\Lambda} \int_{t-\tau}^{t} \mathbf{K}(t-s) x(s) d s\right] .
\end{aligned}
$$

Moreover, based on Lemma 6, we have

$$
\begin{aligned}
& \left.\dot{V}_{2}(t)\right|_{(29)}=\tau \int_{-\tau}^{0}(\mathbf{M K}(0) \mathbf{f}(x(t)))^{\mathrm{T}} \mathbf{Q}(\mathbf{M K}(0) \mathbf{f}(x(t))) d s \\
& -\tau \int_{-\tau}^{0}(\mathbf{M K}(-s) \mathbf{f}(x(t+s)))^{\mathrm{T}} \\
& \times \mathbf{Q}(\mathbf{M K}(-s) \mathbf{f}(x(t+s))) d s \\
& +2 \tau \int_{-\tau}^{0} \int_{t+s}^{t}\left(\mathbf{M} \frac{d \mathbf{K}}{d t}(t-\theta) \mathbf{f}(x(\theta))\right)^{\mathrm{T}} \\
& \times \mathbf{Q}(\mathbf{M K}(t-\theta) \mathbf{f}(x(\theta))) d \theta d s \\
& =\tau \int_{-\tau}^{0}(\mathbf{M K}(0) \mathbf{f}(x(t)))^{\mathrm{T}} \mathbf{Q}(\mathbf{M K}(0) \mathbf{f}(x(t))) d s \\
& -\tau \int_{t-\tau}^{t}(\mathbf{M K}(t-s) \mathbf{f}(x(s)))^{\mathrm{T}} \\
& \times \mathbf{Q}(\mathbf{M K}(t-s) \mathbf{f}(x(s))) d s \\
& -2 \alpha \tau \int_{-\tau}^{0} \int_{t+s}^{t}(\mathbf{M K}(t-\theta) \mathbf{f}(x(\theta)))^{\mathrm{T}} \\
& \times \mathbf{Q}(\mathbf{M K}(t-\theta) \mathbf{f}(x(\theta))) d \theta d s \\
& \leq \tau^{2}(\mathbf{M f}(x(t)))^{\mathrm{T}} \mathbf{K}^{\mathbf{1}^{\mathrm{T}}}(0) \mathbf{Q} \mathbf{K}^{\mathbf{1}}(0) \mathbf{M f}(x(t)) \\
& -\left(\int_{t-\tau}^{t} \mathbf{M K}(t-s) \mathbf{f}(x(s)) d s\right)^{\mathrm{T}} \\
& \times \mathbf{Q}\left(\int_{t-\tau}^{t} \mathbf{M K}(t-s) \mathbf{f}(x(s)) d s\right) .
\end{aligned}
$$

Similarly

$$
\begin{aligned}
\left.\dot{V}_{3}(t)\right|_{(29)} \leq & \tau^{2}(\mathbf{M} x(t))^{\mathrm{T}} \mathbf{K}^{\mathbf{1}^{\mathrm{T}}}(0) \mathbf{R K}^{\mathbf{1}}(0)(\mathbf{M} x(t)) \\
& -\left(\int_{t-\tau}^{t} \mathbf{M K}(t-s) x(s) d s\right)^{\mathrm{T}} \mathbf{R} \\
& \times\left(\int_{t-\tau}^{t} \mathbf{M K}(t-s) x(s) d s\right) .
\end{aligned}
$$


By Assumption $\mathrm{A}_{2}$, it follows that

$$
\begin{aligned}
\mathbf{f}^{\mathrm{T}} & (x(t)) \mathbf{M}^{\mathrm{T}} \mathbf{\Sigma} \mathbf{M f}(x(t)) \\
= & \sum_{j=1}^{m-1}\left[f\left(x_{j}(t)\right)-f\left(x_{j+1}(t)\right)\right]^{\mathrm{T}} \\
& \times \boldsymbol{\Sigma}_{j}\left[f\left(x_{j}(t)\right)-f\left(x_{j+1}(t)\right)\right] \\
\leq & \sum_{j=1}^{m-1}\left[x_{j}(t)-x_{j+1}(t)\right]^{\mathrm{T}} F \boldsymbol{\Sigma}_{j} F\left[x_{j}(t)-x_{j+1}(t)\right] \\
= & x^{\mathrm{T}}(t) \mathbf{M}^{\mathrm{T}} \mathbf{F} \mathbf{\Sigma} \mathbf{F M} x(t)
\end{aligned}
$$

where $\boldsymbol{\Sigma}_{j}=\operatorname{diag}\left(\Sigma_{(j-1) n+1}, \ldots, \Sigma_{j n}\right)$.

Using Lemmas 2 and 5, we obtain

$$
\begin{aligned}
\mathbf{M} \Gamma & =\left(\widetilde{M} \otimes I_{n}\right)(G \otimes \Gamma)=\widetilde{M} G \otimes \Gamma \\
& =\widetilde{H} \widetilde{M} \otimes \Gamma=(\widetilde{H} \otimes \Gamma)\left(\widetilde{M} \otimes I_{n}\right)=\mathbf{H M} \\
\mathbf{M} \boldsymbol{\Lambda} & =\left(\widetilde{M} \otimes I_{n}\right)(G \otimes \Lambda)=\widetilde{M} G \otimes \Lambda \\
& =\widetilde{H} \widetilde{M} \otimes \Lambda=(\widetilde{H} \otimes \Lambda)\left(\widetilde{M} \otimes I_{n}\right)=\overline{\mathbf{H}} \mathbf{M}
\end{aligned}
$$

where $\widetilde{H}=M N_{1} J, \mathbf{H}=\widetilde{H} \otimes \Gamma, \overline{\mathbf{H}}=\widetilde{H} \otimes \Lambda, M$, and $J$ have the same structures as those defined in (22) and (23).

Combining (36)-(41), we obtain

$$
\begin{aligned}
& \left.\dot{V}(t)\right|_{(29)} \leq-2 x^{\mathrm{T}}(t) \mathbf{M}^{\mathrm{T}} \mathbf{P} \mathbf{C}^{\mathbf{1}} \mathbf{M} x(t) \\
& +2 x^{\mathrm{T}}(t) \mathbf{M}^{\mathrm{T}} \mathbf{P} \mathbf{A}^{\mathbf{1}} \mathbf{M} \mathbf{f}(x(t)) \\
& +2 x^{\mathrm{T}}(t) \mathbf{M}^{\mathrm{T}} \mathbf{P} \mathbf{B}^{\mathbf{1}} \mathbf{M} \int_{t-\tau}^{t} \mathbf{K}(t-s) \mathbf{f}(x(s)) d s \\
& +2 x^{\mathrm{T}}(t) \mathbf{M}^{\mathrm{T}} \mathbf{P} \mathbf{H M} x(t) \\
& +2 x^{\mathrm{T}}(t) \mathbf{M}^{\mathrm{T}} \mathbf{P} \overline{\mathbf{H}} \mathbf{M} \int_{t-\tau}^{t} \mathbf{K}(t-s) x(s) d s \\
& +\tau^{2}(\mathbf{M f}(x(t)))^{\mathrm{T}} \mathbf{K}^{\mathbf{1}^{\mathrm{T}}}(0) \mathbf{Q} \mathbf{K}^{\mathbf{1}}(0)(\mathbf{M f}(x(t))) \\
& -\left(\int_{t-\tau}^{t} \mathbf{M K}(t-s) \mathbf{f}(x(s)) d s\right)^{\mathrm{T}} \\
& \times \mathbf{Q}\left(\int_{t-\tau}^{t} \mathbf{M K}(t-s) \mathbf{f}(x(s)) d s\right) \\
& +\tau^{2}(\mathbf{M} x(t))^{\mathrm{T}} \mathbf{K}^{\mathbf{1}^{\mathrm{T}}}(0) \mathbf{R} \mathbf{K}^{\mathbf{1}}(0)(\mathbf{M} x(t))
\end{aligned}
$$

$$
\begin{aligned}
& -\left(\int_{t-\tau}^{t} \mathbf{M K}(t-s) x(s) d s\right)^{\mathrm{T}} \\
& \times \mathbf{R}\left(\int_{t-\tau}^{t} \mathbf{M K}(t-s) x(s) d s\right) \\
& +x^{\mathrm{T}}(t) \mathbf{M}^{\mathrm{T}} \mathbf{F} \boldsymbol{\Sigma} \mathbf{F M} x(t) \\
& -\mathbf{f}^{\mathrm{T}}(x(t)) \mathbf{M}^{\mathrm{T}} \boldsymbol{\Sigma} \boldsymbol{M} f(x(t)) \\
= & \xi^{\mathrm{T}} \mathbf{\Omega} \xi
\end{aligned}
$$

where

$$
\begin{aligned}
\xi= & (\mathbf{M} x(t))^{\mathrm{T}},\left(\int_{t-\tau}^{t} \mathbf{M K}(t-s) x(s) d s\right)^{\mathrm{T}} \\
& \left.(\mathbf{M f}(x(t)))^{\mathrm{T}},\left(\int_{t-\tau}^{t} \mathbf{M K}(t-s) \mathbf{f}(x(s)) d s\right)^{\mathrm{T}}\right)^{\mathrm{T}} .
\end{aligned}
$$

Hence, by Lemma 4 and from (42), we know that under the given condition (30), $\dot{V}(t) \leq 0$ and $\dot{V}(t)=0$ if and only if $\xi \equiv 0$. Moreover, we obtain $V(t) \leq V(0)$, namely, $V(t)$ is a bounded function. Thus, $\|\mathbf{M} x(t)\| \longrightarrow 0$. This completes the proof.

Corollary 1: Under Assumptions $\mathrm{A}_{1}-\mathrm{A}_{4}$, the dynamical system (29) is locally synchronized if there are positive definite matrices $\quad \mathbf{P}=\left(p_{i j}\right) \in R^{(m-1) n \times(m-1) n}, \quad \mathbf{Q}=$ $\left(q_{i j}\right) \in R^{(m-1) n \times(m-1) n}$, and $\mathbf{R}=\left(r_{i j}\right) \in R^{(m-1) n \times(m-1) n}$ and a positive definite diagonal matrix $\boldsymbol{\Sigma}=\operatorname{diag}\left(\Sigma_{1}\right.$, $\left.\Sigma_{2}, \ldots, \Sigma_{(m-1) n}\right) \in R^{(m-1) n \times(m-1) n}$, such as shown at the bottom of the page, where $F=\operatorname{diag}\left(F_{1}, F_{2}, \ldots, F_{n}\right) \in R^{n \times n}$, $\mathbf{F}=E_{m-1} \otimes F, \quad \mathbf{H}=\left(M N_{1} J\right) \otimes \Gamma, \quad \overline{\mathbf{H}}=\left(M N_{1} J\right) \otimes \Lambda$, $M \in R^{(m-1) \times m}$, and $J \in R^{m \times(m-1)}$ have the same structures as those in (22) and (23).

Proof: Instead of using the inequalities (39) and (40), by Assumption $\mathrm{A}_{1}$, it follows that

$$
\begin{aligned}
\mathbf{f}^{\mathrm{T}} & (x(t)) \mathbf{M}^{\mathrm{T}} \mathbf{\Sigma} \mathbf{M f}(x(t)) \\
= & \sum_{j=1}^{m-1}\left[f\left(x_{j}(t)\right)-f\left(x_{j+1}(t)\right)\right]^{\mathrm{T}} \\
& \times \boldsymbol{\Sigma}_{j}\left[f\left(x_{j}(t)\right)-f\left(x_{j+1}(t)\right)\right] \\
\leq & \sum_{j=1}^{m-1}\left[x_{j}(t)-x_{j+1}(t)\right]^{\mathrm{T}} F \boldsymbol{\Sigma}_{j}\left[f\left(x_{j}(t)\right)-f\left(x_{j+1}(t)\right)\right] \\
= & x^{\mathrm{T}}(t) \mathbf{M}^{\mathrm{T}} \mathbf{F} \mathbf{\Sigma} \mathbf{M f}(x(t))
\end{aligned}
$$

where $\boldsymbol{\Sigma}_{j}=\operatorname{diag}\left(\Sigma_{(j-1) n+1}, \ldots, \Sigma_{j n}\right)$.

$$
\boldsymbol{\Omega}=\left(\begin{array}{cccc}
-\mathbf{P C}^{\mathbf{1}}-\mathbf{C}^{\mathbf{1}^{\mathrm{T}}} \mathbf{P}+\mathbf{P} \mathbf{H}+\mathbf{H}^{\mathrm{T}} \mathbf{P}+\tau^{2} \mathbf{K}^{\mathbf{1}^{\mathrm{T}}}(0) \mathbf{R} \mathbf{K}^{\mathbf{1}}(0) & \mathbf{P} \overline{\mathbf{H}} & \mathbf{P A}^{\mathbf{1}}+\mathbf{F} \boldsymbol{\Sigma} & \mathbf{P B}^{\mathbf{1}} \\
\overline{\mathbf{H}^{\mathrm{T}} \mathbf{P}} & -\mathbf{R} & 0 & 0 \\
\mathbf{A}^{\mathbf{1}^{\mathrm{T}} \mathbf{P}+\mathbf{\Sigma} \mathbf{F}} & 0 & \tau^{2} \mathbf{K}^{\mathbf{1}^{\mathrm{T}}}(0) \mathbf{Q} \mathbf{K}^{1}(0)-2 \boldsymbol{\Sigma} & 0 \\
\mathbf{B}^{\mathbf{1}^{\mathrm{T}} \mathbf{P}} & 0 & 0 & -\mathbf{Q}
\end{array}\right)<0
$$


Following the same step as in the proof of Theorem 1, Corollary 1 is proved.

Note that when $m=N$, we can obtain a theorem to ensure the global synchronization of the coupled system (29).

Theorem 2: Under Assumptions $\mathrm{A}_{2}-\mathrm{A}_{4}$, the dynamical system (29) is globally synchronized if there are positive definite matrices $\mathbf{P}=\left(p_{i j}\right) \in R^{(N-1) n \times(N-1) n}, \mathbf{Q}=$ $\left(q_{i j}\right) \in R^{(N-1) n \times(N-1) n}$, and $\mathbf{R}=\left(r_{i j}\right) \in R^{(N-1) n \times(N-1) n}$ and a positive definite diagonal matrix $\boldsymbol{\Sigma}=\operatorname{diag}\left(\Sigma_{1}\right.$, $\left.\Sigma_{2}, \ldots, \Sigma_{(N-1) n}\right) \in R^{(N-1) n \times(N-1) n}$, such that

$$
\left(\begin{array}{cccc}
\Xi & \mathbf{P} \overline{\mathbf{H}} & \mathbf{P A}^{\mathbf{1}} & \mathbf{P B}^{\mathbf{1}} \\
\overline{\mathbf{H}}^{\mathrm{T}} \mathbf{P} & -\mathbf{R} & 0 & 0 \\
\mathbf{A}^{\mathbf{1}^{\mathrm{T}}} \mathbf{P} & 0 & \tau^{2} \mathbf{K}^{\mathbf{1}^{\mathrm{T}}}(0) \mathbf{Q} \mathbf{K}^{\mathbf{1}}(0)-\mathbf{\Sigma} & 0 \\
\mathbf{B}^{\mathbf{1}^{\mathrm{T}}} \mathbf{P} & 0 & 0 & -\mathbf{Q}
\end{array}\right)<0
$$

where $\quad \Xi=-\mathbf{P C}^{\mathbf{1}}-\mathbf{C}^{\mathbf{1}^{\mathrm{T}}} \mathbf{P}+\mathbf{P H}+\mathbf{H}^{\mathrm{T}} \mathbf{P}+\mathbf{F} \boldsymbol{\Sigma} \mathbf{F}+$ $\tau^{2} \mathbf{K}^{\mathbf{1}^{\mathrm{T}}}(0) \mathbf{R K}^{1}(0), \quad F=\operatorname{diag}\left(F_{1}, F_{2}, \ldots, F_{n}\right) \in R^{n \times n}$, $\mathbf{F}=E_{N-1} \otimes F, \quad \mathbf{H}=\left(M N_{1} J\right) \otimes \Gamma, \quad \overline{\mathbf{H}}=\left(M N_{1} J\right) \otimes \Lambda$, $M \in R^{(N-1) \times N}$, and $J \in R^{N \times(N-1)}$ are defined in (22) and (23).

Remark 3: In this paper, only the simple Lyapunov function in (31) is used. It is certain that one can use other complex Lyapunov function candidates to get better conditions for this local synchronization problem by a csimilar approach. Therefore, it is omitted here.

Remark 4: For the Semantic Grid or Virtual Organizations, there are very few models that can be used to investigate their reputation computation. Considering that a node in our new complex network model is a fundamental unit with detailed contents about an entity in the Virtual Organizations, we can use it to study the reputation computation for Virtual Organizations. It is a new method to solve this problem based on our complex network model, which can well describe the dynamics of the reputation degrees of Virtual Organizations.

\section{NUMERICAL EXAMPLE}

In this section, we show one simulation example to illustrate the application of the theoretical results obtained in this paper.

Consider the following reputation computation model:

$$
\begin{aligned}
& \dot{x}_{i}(t)=-C x_{i}(t)+A f\left(x_{i}(t)\right) \\
&+B \int_{t-\tau}^{t} K(t-s) f\left(x_{i}(s)\right) d s+I_{i}(t) \\
&+\sum_{j=1}^{4} G_{i j}\left[\Gamma x_{j}(t)+\Lambda \int_{t-\tau}^{t} K(t-s) x_{j}(s) d s\right] \\
& i=1,2,3,4
\end{aligned}
$$

where

$$
\begin{aligned}
x(t) & =\left(x_{1}(t), x_{2}(t)\right)^{\mathrm{T}} \\
f(x(t)) & =\left(\tanh \left(x_{1}(t)\right), \tanh \left(x_{2}(t)\right)\right)^{\mathrm{T}}
\end{aligned}
$$

$$
\begin{aligned}
& I_{i}(t)=(0.1,0.2)^{\mathrm{T}} \\
& C=\left(\begin{array}{ll}
1 & 0 \\
0 & 1
\end{array}\right), \quad A=\left(\begin{array}{cc}
2.0 & -0.1 \\
-5.0 & 3.0
\end{array}\right) \\
& B=\left(\begin{array}{cc}
-15 & -10 \\
-2 & -45
\end{array}\right), \quad \tau=1 \\
& G=\left(\begin{array}{cccc}
-16 & 15.96 & 0.02 & 0.02 \\
15.96 & -16 & 0.02 & 0.02 \\
0.02 & 0.02 & -0.1 & 0.06 \\
0.02 & 0.02 & 0.06 & -0.1
\end{array}\right) \\
& \Gamma=\left(\begin{array}{ll}
1 & 0 \\
0 & 1
\end{array}\right), \quad \Lambda=\left(\begin{array}{cc}
0.1 & 0 \\
0 & 0.1
\end{array}\right) \\
& K_{i}(s)=\alpha e^{-\alpha s}, \quad \alpha=1, \quad i=1,2 .
\end{aligned}
$$

It is obvious that Assumptions $\mathrm{A}_{1}-\mathrm{A}_{4}$ are satisfied (with $F=E_{2}$ ). Choose the initial conditions

$$
\begin{array}{ll}
x_{1}(s)=\left(\begin{array}{c}
0.1 \\
-0.2
\end{array}\right) & x_{2}(s)=\left(\begin{array}{c}
-0.3 \\
0.4
\end{array}\right) \\
x_{3}(s)=\left(\begin{array}{c}
0.5 \\
-0.6
\end{array}\right) & x_{4}(s)=\left(\begin{array}{c}
-0.7 \\
0.8
\end{array}\right) .
\end{array}
$$

The trajectories of the system (46) are shown in Fig. 1. Here, we consider the local synchronization of the first two nodes in the coupled system (46), i.e., $m=2$. By Theorem 1 and using the linear matrix inequality Matlab Toolbox, the following feasible solutions are obtained:

$$
\begin{aligned}
& \mathbf{P}=\left(\begin{array}{cc}
5.4820 & -0.0304 \\
-0.0304 & 5.0397
\end{array}\right) \\
& \mathbf{Q}=\left(\begin{array}{cc}
77.6007 & 8.0483 \\
8.0483 & 110.9880
\end{array}\right) \\
& \mathbf{R}=\left(\begin{array}{cc}
91.1977 & -10.5443 \\
-10.5443 & 39.3123
\end{array}\right) \\
& \boldsymbol{\Sigma}=\left(\begin{array}{cc}
151.5666 & 0 \\
0 & 135.3840
\end{array}\right) .
\end{aligned}
$$

Based on Theorem 1, the system (46) is locally synchronized. The error distance among the first two nodes in the coupled networks is

$$
\operatorname{err}_{1}(t)=\sum_{i=1}^{2} \sqrt{\left[x_{1 i}(t)-x_{2 i}(t)\right]^{2}}
$$

which is shown in Fig. 2. If we choose $m=4$ and consider the global synchronization of the dynamical system (46), feasible solutions do not exist. The error distance among all four nodes in the coupled network is

$$
\operatorname{err}_{2}(t)=\sum_{i=1}^{2} \sqrt{\sum_{j=2}^{4}\left[x_{1 i}(t)-x_{j i}(t)\right]^{2}}
$$

which is shown in Fig. 3. Therefore, the coupled system (46) is locally synchronized but not globally synchronized. 

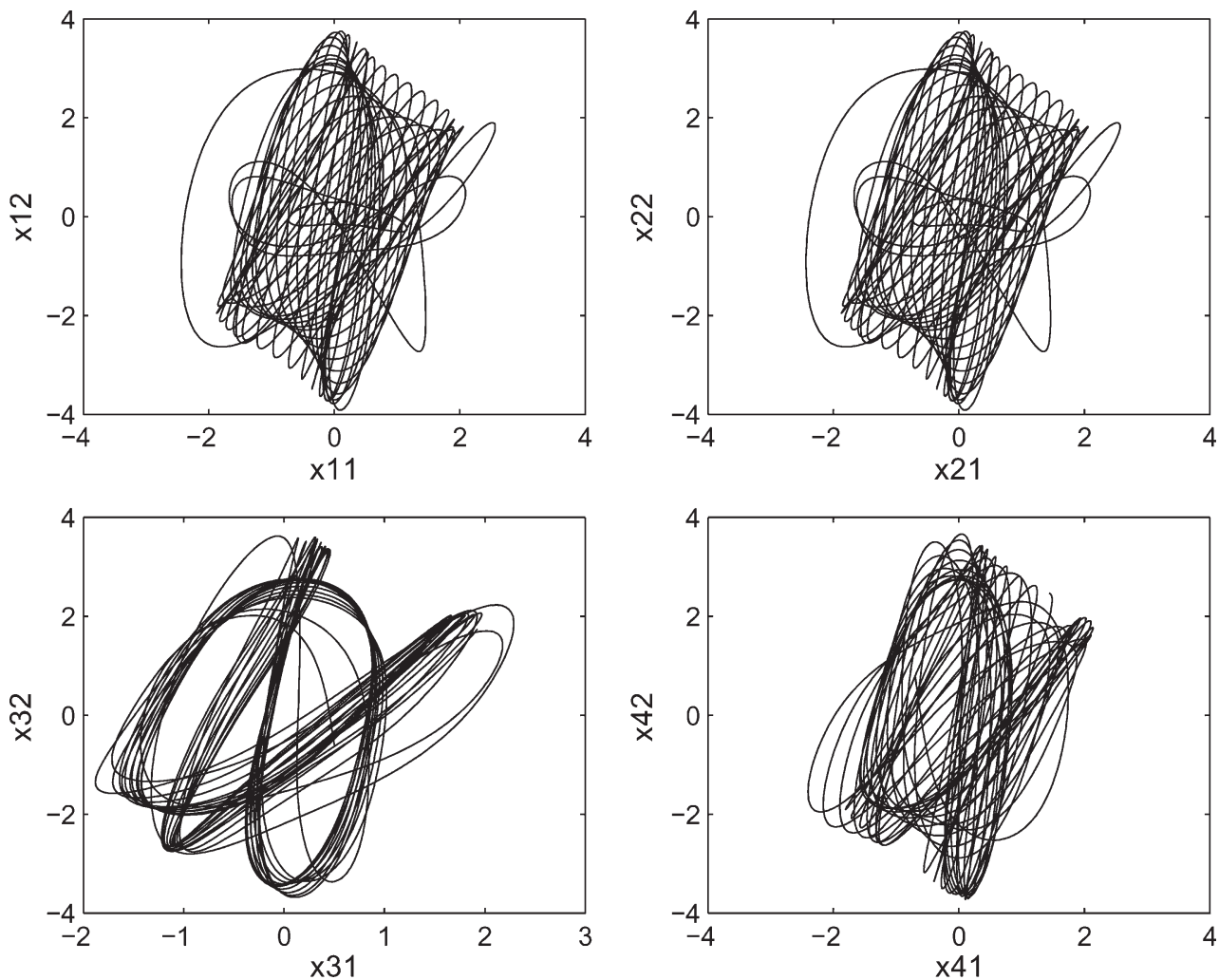

Fig. 1. Trajectories of nodes in the coupled networks (46).
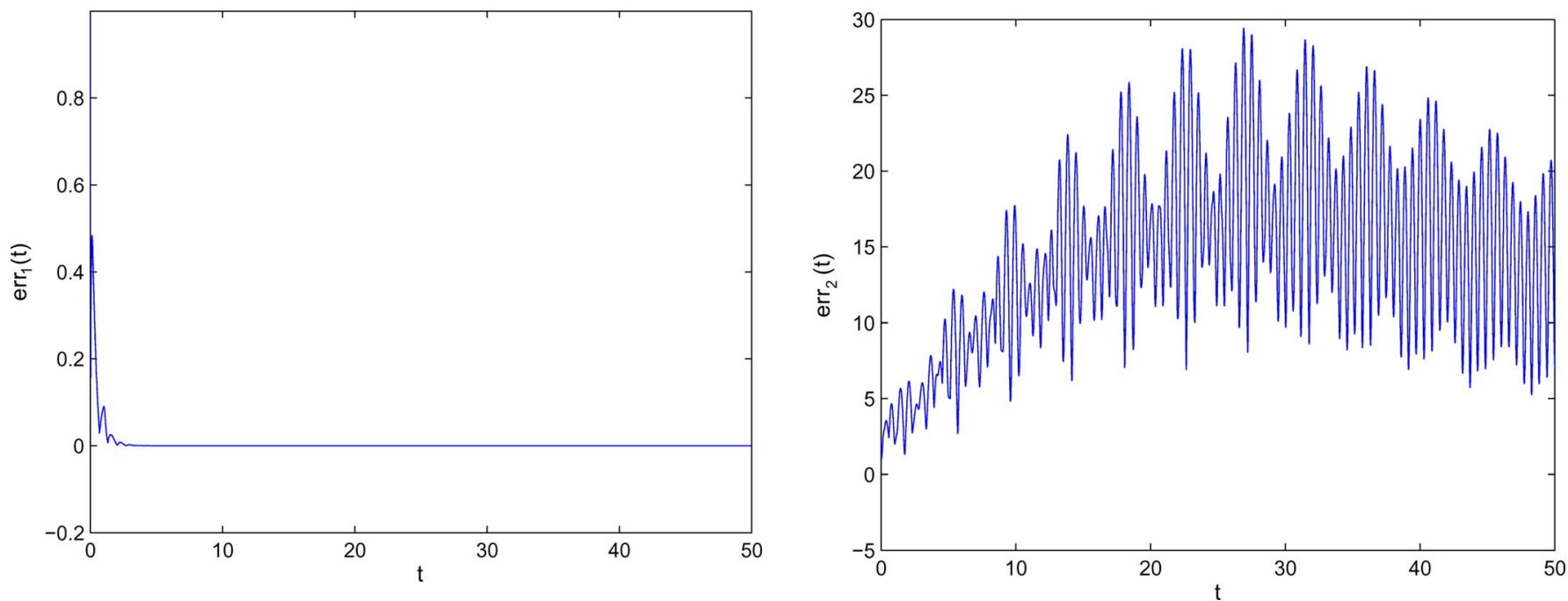

Fig. 2. Error distance of the first two nodes in the coupled network (46).

We may consider nodes 1 and 2 as a group. Their reputation degrees are changed in the same way. In real-world large-scale coupled networks, many nodes in a coupled network can be classified into some groups with similar properties. In this case, one can consider the local synchronization of the reputation computation of the complex network model.

\section{CONCLUSION}

Recently, the Semantic Grid and Virtual Organizations have become focal research topics; however, the corresponding re-

Fig. 3. Error distance of the coupled network (46).

sults, particularly the dynamical models for their reputation dynamics, are still lacking. In this paper, we have built a new complex network model to solve this problem and to study the reputation computation for Virtual Organizations in the sense that a node in the network is a fundamental unit with detailed contents that can present an entity in the Semantic Grid. We have investigated some dynamics of the reputation degrees of entities in Virtual Organizations and their local, as well as global, synchronization.

In this paper, a framework about the local synchronization of a complex network model is established. It is possible to 
extend the obtained results to stochastic networks, discrete-time networks, delayed networks, time-varying networks, and so on. Further research on this new model and the proposed approach will be carried out in the near future.

\section{REFERENCES}

[1] S. H. Strogatz, "Exploring complex networks," Nature, vol. 410, no. 6825, pp. 268-276, Mar. 2001.

[2] D. J. Watts and S. H. Strogatz, "Collective dynamics of 'small-world' networks," Nature, vol. 393, no. 6684, pp. 440-442, Jun. 1998.

[3] X. Wang and G. Chen, "Synchronization in scale-free dynamical networks: Robustness and fragility," IEEE Trans. Circuits Syst. I, Fundam. Theory Appl., vol. 49, no. 1, pp. 54-62, Jan. 2002.

[4] X. Wang and G. Chen, "Synchronization in small-world dynamical networks," Int. J. Bifur. Chaos Appl. Sci. Eng., vol. 12, pp. 187-192, 2002.

[5] C. Wu and L. O. Chua, "Synchronization in an array of linearly coupled dynamical systems," IEEE Trans. Circuits Syst. I, Fundam. Theory Appl., vol. 42, no. 8, pp. 430-447, Aug. 1995.

[6] C. Wu, "Synchronization in arrays of coupled nonlinear systems with delay and nonreciprocal time-varying coupling," IEEE Trans. Circuits Syst. II, Exp. Briefs, vol. 52, no. 5, pp. 282-286, May 2005.

[7] W. Lu and T. Chen, "Synchronization of coupled connected neural networks with delays," IEEE Trans. Circuits Syst. I, Reg. Papers, vol. 51, no. 12, pp. 2491-2503, Dec. 2004.

[8] W. Wang and J. Cao, "Synchronization in an array of linearly coupled networks with time-varying delay," Phys. A, Stat. Mech. Appl., vol. 366, pp. 197-211, Jul. 2006.

[9] J. Cao, P. Li, and W. Wang, "Global synchronization in arrays of delayed neural networks with constant and delayed coupling," Phys. Lett. A, vol. 353, no. 4, pp. 318-325, May 2006.

[10] C. Li and G. Chen, "Stability of a neural network model with small-world connections," Phys. Rev. E, Stat. Phys. Plasmas Fluids Relat. Interdiscip. Top., vol. 68, no. 5, p. 052 901, Nov. 2003.

[11] G. Chen, J. Zhou, and Z. Liu, "Global synchronization of coupled delayed neural networks and applications to chaotic CNN models," Int. J. Bifur. Chaos Appl. Sci. Eng., vol. 14, no. 7, pp. 2229-2240, 2004

[12] Z. Li and G. Chen, "Global synchronization and asymptotic stability of complex dynamical networks," IEEE Trans. Circuits Syst. II, Exp. Briefs, vol. 53 , no. 1 , pp. $28-33$, Jan. 2006

[13] C. Li, S. Li, X. Liao, and J. Yu, "Synchronization in coupled map lattices with small-world delayed interactions," Phys. A, Stat. Mech. Appl., vol. 335, no. 3/4, pp. 365-370, Apr. 2004.

[14] C. Li, H. Xu, X. Liao, and J. Yu, "Synchronization in small-world oscillator networks with coupling delays," Phys. A, Stat. Mech. Appl., vol. 335, no. 3/4, pp. 359-364, Apr. 2004.

[15] C. Li and G. Chen, "Synchronization in general complex dynamical networks with coupling delays," Phys. A, Stat. Mech. Appl., vol. 343, pp. 263-278, Nov. 2004.

[16] J. Lü, X. Yu, and G. Chen, "Chaos synchronization of general complex dynamical networks," Phys. A, Stat. Mech. Appl., vol. 334, no. 1/2, pp. 281302, Mar. 2004.

[17] Z. Li and G. Chen, "Robust adaptive synchronization of uncertain dynamical networks," Phys. Lett. A, vol. 324, no. 2/3, pp. 166-178, Apr. 2004.

[18] A. Pogromsky, G. Santoboni, and H. Nijmeijer, "Partial synchronization: from symmetry towards stability," Physica, D, vol. 172, no. 1, pp. 65-87, Nov. 2002.

[19] S. Yanchuk, Y. Maistrenko, and E. Mosekilde, "Partial synchronization and clustering in a system of diffusively coupled chaotic oscillators," Math. Comput. Simul., vol. 54, no. 6, pp. 491-508, Jan. 2001.

[20] A. Lipowski and M. Droz, "Synchronization and partial synchronization of linear maps," Phys. A, Stat. Mech. Appl., vol. 347, pp. 38-50, Mar. 2005.

[21] R. A. Horn and C. R. Johnson, Topics in Matrix Analysis. Cambridge, U.K.: Cambridge Univ. Press, 1991.

[22] K. Q. Gu, V. L. Kharitonov, and J. Chen, Stability of Time-Delay Systems. Cambridge, MA: Birkhauser Boston, 2003.

[23] F. Azzedin and M. Maheswaran, "Integrating trust into grid resource management systems," in Proc. Int. Conf. Parallel Process., 2002, pp. 1-8.

[24] D. Roure, N. R. Jennings, and N. R. Shadbolt, "The Semantic Grid: Past, present, and future," Proc. IEEE, vol. 93, no. 3, pp. 669-681, Mar. 2005.

[25] X. Gui, B. Xie, Y. Li, and D. Qian, "Study on the behavior-based trust model in grid security system," in Proc. IEEE Int. Conf. Service Comput., 2004, pp. 506-509.
[26] Y. Lee, "A dynamic virtual organization solution for web-services based grid middleware," in Proc. 16th Int. Workshop Database Expert Syst. Appl., Aug. 2005, pp. 40-44.

[27] M. Niinimaki, J. White, W. Cerff, and J. Hahala, "Using virtual organizations membership system with EDG's grid security and database acess," in Proc. 15th Int. Workshop Database Expert Syst. Appl., Sep. 2004, pp. 517-522.

[28] C. Lin, V. Varadharajan, and Y. Wang, "Enhancing grid security with trust management," in Proc. IEEE Int. Conf. Service Comput., Sep. 2004, pp. 303-310.

[29] "Semantics, resource and grid," Future Gener. Comput. Syst., vol. 20, no. 1, pp. 1-5, Jan. 2004.

[30] D. J. Watts, Small-Worlds: The Dynamics of Networks Between Order and Randomness. Princeton, NJ: Princeton Univ. Press, 1999.

[31] J. Golbeck and J. Hendler, "Inferring reputation on the semantic web," in Proc. 13th Int. Web Conf. WWW, New York, May 17-22, 2004.

[32] C. Goble, R. Stevens, and S. Bechhofer, "The semantic web and knowledge grids," Drug Discov. Today-Technol., vol. 2, no. 3, pp. 225-233, 2005.

[33] D. Bell, C. Bussler, and J. Yang, "The semantic web and web services," Inf. Syst., vol. 31, no. 4/5, pp. 229-231, Jun./Jul. 2006.

[34] S. Agarwal, S. Handschuh, and S. Staab, "Annotation, composition and invocation of semantic web services," J. Web Semant., vol. 2, no. 1, pp. 31-48, Dec. 2004.

[35] J. Cao, W. Yu, and Y. Qu, "A new complex network model and convergence dynamics for reputation computation in virtual organizations," Phys. Lett. A, vol. 356, no. 6, pp. 414-425, Aug. 2006.

[36] W. Yu and J. Cao, "Adaptive Q-S (lag, anticipated, and complete) time-varying synchronization and parameters identification of uncertain delayed neural networks," Chaos, vol. 16, no. 2, p. 023119 , Jun. 2006.

[37] J. Zhou, J. A. Lu, and J. Lü, "Adaptive synchronization of an uncertain complex dynamical network," IEEE Trans. Autom. Control, vol. 51, no. 4, pp. 652-656, Apr. 2006.

[38] J. Lü and G. Chen, "A time-varying complex dynamical network models and its controlled synchronization criteria," IEEE Trans. Autom. Control, vol. 50, no. 6, pp. 841-846, Jun. 2005.

[39] J. Lü, X. Yu, G. Chen, and D. Cheng, "Characterizing the synchronizability of small-world dynamical networks," IEEE Trans. Circuits Syst. I, Reg. Papers, vol. 51, no. 4, pp. 787-796, Apr. 2004.

[40] W. Yu and J. Cao, "Synchronization control of stochastic delayed neural networks," Phys. A, Stat. Mech. Appl., vol. 373, pp. 252-260, Jan. 2007

[41] W. Yu, J. Cao, and G. Chen, "Robust adaptive control of unknown modified Cohen-Grossberg neural networks with delay," IEEE Trans. Circuits Syst. II, Analog Digit. Signal Process., vol. 54, no. 6, pp. 502-506, Jun. 2007.

[42] W. Yu, G. Chen, J. Cao, J. Lü, and U. Parlitz, "Parameter identification of dynamical systems from time series," Phys. Rev. E, Stat. Phys. Plasmas Fluids Relat. Interdiscip. Top., vol. 75, no. 6, p. 067201 , Jun. 2007.

[43] W. Yu, J. Cao, K. W. Wong, and J. Lü, "New communication schemes based on adaptive synchronization," Chaos, vol. 17, no. 3, p. 033114 , Sep. 2007.

[44] W. Yu, J. Cao, and J. Lü, "Global synchronization of linearly hybrid coupled networks with time-varying delay," SIAM J. Appl. Dyn. Syst., vol. 7, no. 1, pp. 108-133, Jan. 2008.

[45] J. Cao, G. Chen, and P. Li, "Global synchronization in an array of delayed neural networks with hybrid coupling," IEEE Trans. Syst., Man, Cybern. B, Cybern., vol. 38, no. 2, pp. 488-498, Apr. 2008.

[46] C. Cheng, T. Liao, J. Yan, and C. Hwang, "Exponential synchronization of a class of neural networks with time-varying delays," IEEE Trans. Syst., Man, Cybern. B, Cybern., vol. 36, no. 1, pp. 209-215, Feb. 2006.

[47] R. Palm, "Synchronization of decentralized multiple-model systems by market-based optimization," IEEE Trans. Syst., Man, Cybern. B, Cybern., vol. 34, no. 1, pp. 665-671, Feb. 2004.

[48] V. Gazi, "Stability of an asynchronous swarm with time-dependent communication links," IEEE Trans. Syst., Man, Cybern. B, Cybern., vol. 38, no. 1, pp. 267-274, Feb. 2008.

[49] J. Liang, Z. Wang, and X. Liu, "Exponential synchronization of stochastic delayed discrete-time complex networks," Nonlinear Dyn., vol. 53, no. $1 / 2$, pp. $153-165,2008$.

[50] J. Liang, Z. Wang, Y. Liu, and X. Liu, "Global synchronization control of general delayed discrete-time networks with stochastic coupling and disturbances," IEEE Trans. Syst., Man, Cybern. B, Cybern., vol. 38, no. 4, pp. 1073-1083, Aug. 2008. 


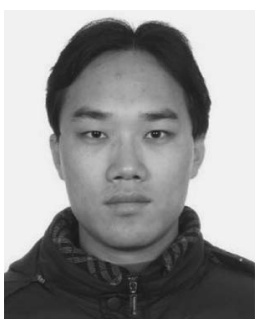

Wenwu Yu (S'07) received the B.S. degree in information and computing science and the M.S. degree in applied mathematics from the Department of Mathematics, Southeast University, Nanjing, China, in 2004 and 2007, respectively. He is currently working toward the Ph.D. degree in the Department of Electronic Engineering, City University of Hong Kong, Kowloon, Hong Kong.

From November 2006 to January 2007, he was a Research Assistant with the Department of Electronic Engineering, City University of Hong Kong. From July 2007 to October 2007, he was a Research Assistant with the Department of Electrical Engineering, Columbia University in the City of New York, New York. From November 2007 to January 2008, he was a Visiting Scholar with the Institute of System Science, Academy of Mathematics and System Science, Chinese Academy of Sciences, Beijing, China. He is the author or coauthor of about 20 journal papers and a reviewer of several journals. His research interests include stability theory, bifurcation analysis, chaos synchronization and control, complex networks and systems, stochastic systems, neural networks, cryptography, communications, and multiagent systems.

$\mathrm{Mr}$. Yu is the recipient of the Best Master Degree Theses Award from Jiangsu Province, China, in 2008.

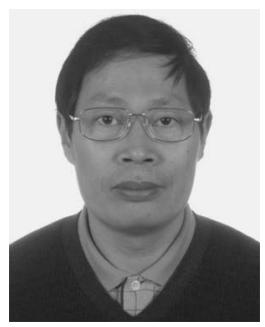

Jinde Cao (M'07-SM'07) received the B.S. degree in mathematics/applied mathematics from Anhui Normal University, Wuhu, China, in 1986, the M.S. degree in mathematics/applied mathematics from Yunnan University, Kunming, China, in 1989, and the $\mathrm{Ph} . \mathrm{D}$. degree in mathematics/applied mathematics from Sichuan University, Chengdu, China, in 1998.

From March 1989 to May 2000, he was with Yunnan University, Kunming, China, where he was a Professor from 1996 to 2000. Since May 2000, he has been with the Department of Mathematics, Southeast University, Nanjing, China, where he is currently a TePin Professor and a Doctoral Advisor. From July 2001 to June 2002, he was a Postdoctoral Research Fellow with the Department of Automation and Computer-aided Engineering, Chinese University of Hong Kong, Shatin, Hong Kong. In 2006, 2007, and 2008, he was a Visiting Research Fellow or a Visiting Professor with the School of Information Systems, Computing, and Mathematics, Brunel University, Uxbridge, U.K. He is the author or coauthor of more than 160 journal papers and five edited books and is a reviewer of Mathematical Reviews and Zentralblatt-Math. His research interests include nonlinear systems, neural networks, complex systems and complex networks, stability theory, and applied mathematics.

Prof. Cao is an Associate Editor of the IEEE TRANSACTION ON NEURAL NETWORKS, Journal of the Franklin Institute, Mathematics, and Computers in Simulation, and Neurocomputing.

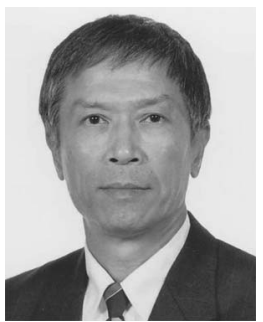

Guanrong Chen (M'89-SM'92-F'97) received the M.Sc. degree in computer science from the Sun Yat-Sen University, Guangzhou, China, in 1981 and the Ph.D. degree in applied mathematics from Texas A\&M University, College Station, in 1987.

He was a tenured Full Professor with the University of Houston, TX, and is currently a Chair Professor and the Founding Director of the Centre for Chaos and Complex Networks, City University of Hong Kong, Kowloon, Hong Kong, where he is also currently with the Department of Electronic Engineering. He is an Honorary Professor at different ranks in more than 20 universities in Argentina, Australia, China, and USA.

Prof. Chen served and is serving as Chief Editor, Deputy Chief Editor, Advisory Editor, Features Editor, and Associate Editor for eight international journals, including the IEEE TRANSACTIONS ON CIRCUITS AND SYSTEMS, IEEE TRANSACTIONS ON AUTOMATIC CONTROL, and the International Journal of Bifurcation and Chaos. He is the recipient of the 1998 Harden-Simons Prize for the Outstanding Journal Paper Award from the American Society of Engineering Education, the $2001 \mathrm{M}$. Barry Carlton Best Transactions Paper Award from the IEEE Aerospace and Electronic Systems Society, the 2002 Best Paper Award from the Institute of Information Theory and Automation, Academy of Science of the Czech Republic, and the 2005 IEEE GuilleminCauer Best Transaction Paper Award from the Circuits and Systems Society.

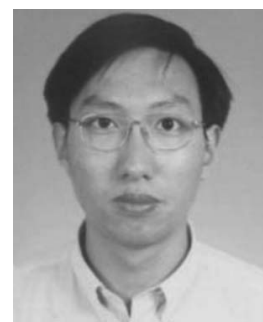

Jinhu Lü (M'03-SM'06) received the Ph.D. degree in applied mathematics from the Chinese Academy of Sciences, Beijing, China, in 2002.

$\mathrm{He}$ held several visiting positions in Australia, Canada, France, Germany, Hong Kong, and USA and was a Visiting Fellow with Princeton University, Princeton, NJ, from 2005 to 2006 . He is currently an Associate Professor with the Institute of Systems Science, Academy of Mathematics and Systems Science, Chinese Academy of Sciences. He is the author of two research monographs and more than 70 international journal papers published in the fields of nonlinear circuits and systems, complex networks, and complex systems and is the coauthor of the Most Cited Science Citation Index Paper of Chinese Scholars in the field of mathematics during the periods of 2001-2005 and 2002-2006.

Dr. Lü is the recipient of the prestigious Presidential Outstanding Research Award from the Chinese Academy of Sciences in 2002, the National Best Ph.D. Theses Award from the Office of Academic Degrees Committee of the State Council and the Ministry of Education of China in 2004, the First Prize of Natural Science Award from the Ministry of Education of China in 2007, and the Lu Jiaxi Youth Talent Award from the Chinese Academy of Sciences in 2008. He served as a member in the Technical Committees of several international conferences and is now serving as a member in the Technical Committees of Nonlinear Circuits and Systems and of Neural Systems and Applications in the IEEE Circuits and Systems Society. He is also an Associate Editor of IEEE TRANSACTIONS ON CIRCUITS AND SYSTEMS II, Journal of Systems Science and Complexity, and Dynamics of Continuous, Discrete and Impulsive Systems Series A, Mathematical Analys.

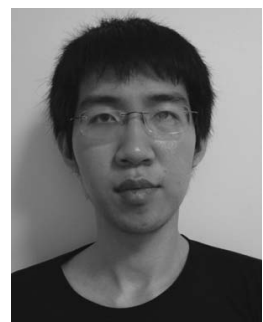

Jian Han (S'08) received the B.S. degree in electrical engineering from the Department of Electronic Engineering, Tsinghua University, Beijing, China, in 2007. He is currently working toward the Ph.D. degree in electrical engineering in the School of Engineering and Applied Sciences, Harvard University, Cambridge, MA.

His research interests include mixed-signal very large integration design, dynamic RAM circuit design, and high-speed communication circuit design.

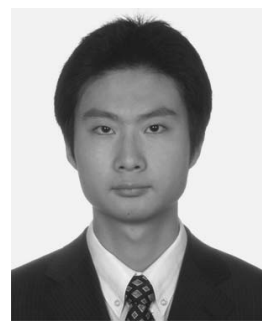

Wei Wei received the B.S. degree in information engineering from the School of Information Science and Engineering, Southeast University, Nanjing, China, in 2006. He is currently working toward the M.S. degree in the Department of Electrical Engineering, Stanford University, Stanford, CA.

His research interests include complex networks and systems, optimization, and communication systems. 